\begin{tabular}{|c|l|}
\hline Title & Tunable phi Josephson junction with a quantum anomal ous Hall insulator \\
\hline Author(s) & Sakurai, Keimei; I kegaya, Satoshi; A sano, Y asuhiro \\
\hline Citation & $\begin{array}{l}\text { Physical Review B, 96(22), 224514 } \\
\text { https://doi.org/10.1103/PhysRevB.96.224514 }\end{array}$ \\
\hline Issue Date & 2017-12-27 \\
\hline Doc URL & http://hdl.handle.net/2115/68257 \\
\hline Rights & ○2017 A merican Physical Society \\
\hline Type & article \\
\hline File Information & PhysRevB.96.224514.pdf \\
\hline
\end{tabular}

Instructions for use 


\title{
Tunable- $\varphi$ Josephson junction with a quantum anomalous Hall insulator
}

\author{
Keimei Sakurai, ${ }^{1}$ Satoshi Ikegaya, ${ }^{1}$ and Yasuhiro Asano ${ }^{1,2,3}$ \\ ${ }^{1}$ Department of Applied Physics, Hokkaido University, Sapporo 060-8628, Japan \\ ${ }^{2}$ Center for Topological Science and Technology, Hokkaido University, Sapporo 060-8628, Japan \\ ${ }^{3}$ Moscow Institute of Physics and Technology, 141700 Dolgoprudny, Russia
}

(Received 7 September 2017; published 27 December 2017)

\begin{abstract}
We theoretically study the Josephson current in a superconductor/quantum anomalous Hall insulator/superconductor junction by using the lattice Green function technique. When an in-plane external Zeeman field is applied to the quantum anomalous Hall insulator, the Josephson current $J$ flows without a phase difference across the junction $\theta$. The phase shift $\varphi$ appearing in the current-phase relationship $J \propto \sin (\theta-\varphi)$ is proportional to the amplitude of Zeeman fields and depends on the direction of Zeeman fields. A phenomenological analysis of the Andreev reflection processes explains the physical origin of $\varphi$. In a quantum anomalous Hall insulator, time-reversal symmetry and mirror-reflection symmetry are broken simultaneously. However, magnetic mirror-reflection symmetry is preserved. Such characteristic symmetry properties enable us to have a tunable $\varphi$ junction with a quantum Hall insulator.
\end{abstract}

DOI: 10.1103/PhysRevB.96.224514

\section{INTRODUCTION}

The Josephson effect is a macroscopic quantum phenomenon caused by the spatial fluctuations of a superconducting phase [1]. When two superconductors (S) sandwich a material $\mathrm{X}$, the Josephson current $J$ flows as a function of the phase difference across the junction $\theta$. The currentphase $(J-\theta)$ relationship (CPR) reflects well the electronic properties of $X[2,3]$. When $X$ is an insulator, the CPR is sinusoidal $J=J_{0} \sin \theta$ with $J_{0}>0$ being the critical current [4]. Such a junction is called 0 junction because the junction energy is minimum at the zero phase difference. A $\pi$ junction in which the energy is minimum at $\theta= \pm \pi$ can be realized when we choose a ferromagnet as $X[5,6]$. The spin-singlet pairing correlation spatially oscillates and changes its sign under the exchange potential. Therefore, such a Josephson junction undergoes the transition between the 0 state and the $\pi$ state alternatively as the variation of the thickness in the ferromagnet $[7,8]$. In the view of the device application, a $\pi$ junction plays a crucial role in constructing a flux qubit. Choosing an insulating ferromagnet as $\mathrm{X}$ makes a device with a long coherence time possible [9].

The energy of a Josephson junction some of the time takes its minimum at a phase difference $\varphi$, which is neither 0 nor $\pi$. The CPR in such $\varphi$ junction $J=J_{0} \sin (\theta-\varphi)$ suggests that the current flows even at the zero phase difference [10-12]. Breaking time-reversal symmetry in $\mathrm{X}$ is a necessary condition to realize the $\varphi$ junction. The value of $\varphi$ is determined by characteristic electronic structures in X. So far, the possibility of $\varphi$ junction has been discussed theoretically in various Josephson junctions with $\mathrm{X}$ being multilayered ferromagnets [13,14], quantum point contacts [15], quantum dots [16-18], nanowires [11,19], topological materials [20,21], and a ferromagnet without inversion symmetry [22]. In experiments, on the other hand, the realization of a $\varphi$ junction has been reported only in a Josephson junction with a nanowire quantum dot [23]. At present, it is not easy to control the phase shift $\varphi$ after fabricating Josephson junctions.

In this paper, we study the Josephson effect in superconductor/quantum anomalous Hall insulator/superconductor
(S/QAHI/S) junctions theoretically. A QAHI is a topologically nontrivial material in two dimensions and breaks time-reversal symmetry by its spontaneous magnetization. In experiments, doping of magnetic elements such as $\mathrm{Cr}$ [24-27] and V [28] onto a thin film of a topological insulator $(\mathrm{Bi}, \mathrm{Sb})_{2} \mathrm{Te}_{3}$ enables QAHIs. According to the bulk-boundary correspondence, nonzero Chern number implies the presence of chiral edge states. We will discuss characteristic features in the Josephson current flowing through such a chiral edge channel. The Josephson current is calculated numerically by using the lattice Green function method. When we apply an in-plane external Zeeman field to QAHI, the junction becomes a $\varphi$ junction. Moreover, the value of $\varphi$ is proportional to a Zeeman field, which suggests a possibility of a tunable $\varphi$ junction. A phenomenological argument explains well the physical origin of the $\varphi$ junction. The breaking magnetic mirror reflection symmetry (MMRS) of the Hamiltonian is a key property to understand the physics behind the phase shift $\varphi$. We also demonstrate that random impurities and the asymmetry of junction geometry in real space break MMRS and make S/QAHI/S be a $\varphi$ junction.

This paper is organized as follows. In Sec. II, we show the Hamiltonian of a QAHI on a tight-binding model and discuss the numerical results of the Josephson current. In Sec. III, the mechanism of the $\varphi$ phase shift in the CPR is explained by a phenomenological analysis of the Andreev reflection processes. The numerical results in the presence of random impurities and junction asymmetry are presented in Sec. IV. The conclusion is given in Sec. V.

\section{NUMERICAL RESULTS ON A TIGHT-BINDING MODEL}

Let us consider a S/QAHI/S junction on a two-dimensional tight-binding model as shown in Fig. 1. Throughout this paper, we measure the length in unit of the lattice constant. A vector $\boldsymbol{r}=j \boldsymbol{x}+m \boldsymbol{y}$ points on a lattice site, where $\boldsymbol{x}$ and $\boldsymbol{y}$ are the unit vectors in the $x$ and $y$ directions, respectively. The junction consists of three regions: a quantum anomalous hall insulator (i.e., $1 \leqslant j \leqslant L$ ) and two superconductors (i.e., $-\infty \leqslant j \leqslant 0$ 


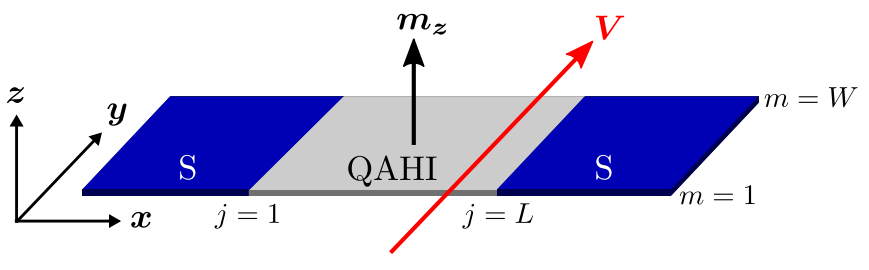

FIG. 1. Schematic figure of a Josephson junction with a QAHI. The magnetic moment $\boldsymbol{m}_{\mathbf{z}}$ points in the $z$ direction. An external Zeeman field $\boldsymbol{V}$ is applied within the two-dimensional plane.

and $L+1 \leqslant j \leqslant \infty)$. An external Zeeman field $\boldsymbol{V}$ is applied in the QAHI segment. The width of the junction is $W$. We apply the hard wall boundary condition in the $y$ direction. The Hamiltonian of the junction is given by

$$
\mathcal{H}=\mathcal{H}_{\mathrm{L}}+\mathcal{H}_{\mathrm{QAHI}}+\mathcal{H}_{\mathrm{R}}
$$

The first and the third term in Eq. (1) are the Hamiltonians of an $s$-wave superconductor on the left and that on the right, respectively. They are given by

$$
\begin{gathered}
\mathcal{H}_{\mathrm{L}(\mathrm{R})}=\sum_{\boldsymbol{r}, \boldsymbol{r}^{\prime}} \Psi_{\boldsymbol{r}}^{\dagger}\left[\begin{array}{cc}
\hat{h}_{\boldsymbol{r}, \boldsymbol{r}^{\prime}} & \hat{\Delta}_{\boldsymbol{r}, \boldsymbol{r}^{\prime}} \mathrm{e}^{i \theta_{\mathrm{L}(\mathrm{R})}} \\
-\hat{\Delta}_{\boldsymbol{r}, \boldsymbol{r}^{\prime}} \mathrm{e}^{-i \theta_{\mathrm{L}(\mathrm{R})}} & -\hat{h}_{\boldsymbol{r}, \boldsymbol{r}^{\prime}}^{*}
\end{array}\right] \Psi_{\boldsymbol{r}^{\prime}}, \\
\hat{h}_{\boldsymbol{r}, \boldsymbol{r}^{\prime}}=\left[-t \delta_{\left|\boldsymbol{r}-\boldsymbol{r}^{\prime}\right|, 1}+\left(4 t-\mu_{\mathrm{s}}\right) \delta_{\boldsymbol{r}, \boldsymbol{r}^{\prime}}\right] \hat{\sigma}_{0}, \\
\hat{\Delta}_{\boldsymbol{r}, \boldsymbol{r}^{\prime}}=i \Delta \hat{\sigma}_{2} \delta_{\boldsymbol{r}, \boldsymbol{r}^{\prime}}, \\
\Psi_{\boldsymbol{r}}=\left(c_{\boldsymbol{r}, \uparrow}, c_{\boldsymbol{r}, \downarrow}, c_{\boldsymbol{r}, \uparrow}^{\dagger}, c_{\boldsymbol{r}, \downarrow}^{\dagger}\right)^{\mathrm{T}},
\end{gathered}
$$

where $c_{r, \sigma}^{\dagger}\left(c_{r, \sigma}\right)$ is the creation (annihilation) operator of an electron at $\boldsymbol{r}$ with spin $\sigma(=\uparrow$ or $\downarrow), \hat{\sigma}_{j}$ with $j=1-3$ are the Pauli matrices in spin space, $\hat{\sigma}_{0}$ is the unit matrix, $\mu_{\mathrm{s}}$ is the chemical potential in the superconductors, $\mathrm{T}$ means the transpose of a matrix, and $\Delta$ is the amplitude of the pair potential. We consider the hopping integral $t$ between the nearest-neighbor sites. The phase of the left (right) superconductor is $\theta_{L}\left(\theta_{R}\right)$. The physical values depend only on the phase difference across the junction $\theta=\theta_{L}-\theta_{R}$.

The second term in Eq. (1) indicates the Hamiltonian of a QAHI [29]:

$$
\begin{aligned}
\mathcal{H}_{\mathrm{QAHI}}= & \sum_{\boldsymbol{r}, \boldsymbol{r}^{\prime}} \Psi_{\boldsymbol{r}}^{\dagger}\left[\begin{array}{cc}
\hat{Q}_{\boldsymbol{r}, \boldsymbol{r}^{\prime}} & 0 \\
0 & -\hat{Q}_{\boldsymbol{r}, \boldsymbol{r}^{\prime}}^{*}
\end{array}\right] \Psi_{\boldsymbol{r}^{\prime}}, \\
\hat{Q}_{\boldsymbol{r}, \boldsymbol{r}^{\prime}}= & {\left[-t \delta_{\left|\boldsymbol{r}-\boldsymbol{r}^{\prime}\right|, 1}+\left(4 t-m_{\mathrm{z}}\right) \delta_{\boldsymbol{r}, \boldsymbol{r}^{\prime}}\right] \hat{\sigma}_{3} } \\
& -\frac{i \lambda}{2}\left[\delta_{j, j^{\prime}+1}-\delta_{j+1, J^{\prime}}\right] \delta_{m, m^{\prime}} \hat{\sigma}_{2} \\
& +\frac{i \lambda}{2}\left[\delta_{m, m^{\prime}+1}-\delta_{m+1, m^{\prime}}\right] \delta_{j, j^{\prime}} \hat{\sigma}_{1} \\
& -V_{x} \delta_{\boldsymbol{r}, \boldsymbol{r}^{\prime}} \hat{\sigma}_{1}-V_{y} \delta_{\boldsymbol{r}, \boldsymbol{r}^{\prime}} \hat{\sigma}_{2},
\end{aligned}
$$

where $\lambda$ is the amplitude of the spin-orbit interaction and $m_{\mathrm{z}}$ is a Zeeman potential induced by the spontaneous magnetization. For $m_{\mathrm{z}}>0$, a QAHI has a chiral edge state characterized by a Chern number of $\mathbb{Z}=-1$ [30]. The insulating gap can be described by these parameters as $E_{\mathrm{g}} \sim 2 \lambda \sqrt{m_{\mathrm{z}} / t}$. (see also Appendix A for details.) In addition to the spontaneous magnetic moment in the $z$ direction, we consider Zeeman potentials $V_{x}$ in the $x$ direction and $V_{y}$ in the $y$ direction by applying an external magnetic field. In what follows, we assume weak Zeeman field so that $\left|V_{x}\right| \ll m_{z}$ and $\left|V_{y}\right| \ll m_{z}$ are satisfied.

We calculate the Josephson current based on the current formula [31-33]

$$
\begin{gathered}
J=\frac{i e}{2 \hbar} T \sum_{\omega_{n}} \operatorname{Tr}\left[\check{\tau}_{3} \check{T}_{+} \breve{G}\left(\boldsymbol{r}, \boldsymbol{r}+\boldsymbol{x} ; \omega_{n}\right)-\check{\tau}_{3} \check{T}_{-} \breve{G}\left(\boldsymbol{r}+\boldsymbol{x}, \boldsymbol{r} ; \omega_{n}\right)\right] \\
\check{T}_{ \pm}=\left[\begin{array}{cc}
\hat{t}_{ \pm} & 0 \\
0 & -\hat{t}_{ \pm}^{*}
\end{array}\right], \quad \hat{t}_{ \pm}=\left[\begin{array}{cc}
-t \overline{1} & \mp \frac{\lambda}{2} \overline{1} \\
\pm \frac{\lambda}{2} \overline{1} & t \overline{1}
\end{array}\right],
\end{gathered}
$$

where $\breve{G}\left(\boldsymbol{r}, \boldsymbol{r}^{\prime} ; \omega_{n}\right)$ is the Matsubara Green function and $\omega_{n}=$ $(2 n+1) \pi k_{B} T$ is the Matsubara frequency with $n, T$ and $k_{B}$ being an integer number, a temperature and the Boltzmann constant, respectively. The Green function is calculated numerically by using the lattice Green function technique [34]. In the above equations, $\hat{\therefore} \cdot(. \therefore)$ indicates $2 W \times 2 W$ $(4 W \times 4 W)$ matrices, and the $W \times W$ unit matrix is denoted by $\overline{1}$. In Eq. (8), $\check{\tau}_{3}$ is the third Pauli matrix in particle-hole space, and Tr means the trace over spin space, particle-hole space, and the summation over the lattice sites in the $y$ direction.

Throughout this paper, we fix basic parameters as $m_{z}=$ $1.5 t$ and $\lambda=1.0 t$. The chiral edge states spatially localize within two lattice constants from the surface under these parameter choices. The size of a QAHI should be much larger than the localization length. Therefore, we choose $L=80$ and $W=20$. The chemical potential in two superconductors is chosen as $\mu_{s}=2.7 t$. We find that $\mu_{s}>m_{z}$ is necessary so that the normal conductance through the chiral edge is quantized at $e^{2} / h$. Otherwise, the normal conductance becomes smaller than $e^{2} / h$. The amplitude of the pair potential at the zero temperature $\Delta_{0}=0.001 t$ is much smaller than $\mu_{s}$. We mainly calculate the Josephson current at $T=0.1 T_{c}$, where $T_{c}$ is the transition temperature. We assume that the superconducting gap is smaller than the insulating gap in a QAHI (i.e., $\Delta_{0} \ll E_{\mathrm{g}}$ ). As a result, the Josephson current flows only through the chiral edge states in a QAHI.

In Fig. 2(a), we first discuss the CPR in the absence of an external Zeeman field as shown with a solid line. The Josephson currents are normalized to $J_{0} \times 10^{-3}$ with $J_{0}=e \Delta_{0} / \hbar$. The CPR at $V_{y}=0$ in Fig. 2(a) is sinusoidal, which is a robust feature independent of parameters such as $T, \mu_{s}$, and $m_{z}$. In Appendix B, we demonstrate the CPR for several choices of $\mu_{s}$ to check the robustness of 0 junction in the absence of the Zeeman field. Although the dependence of the Josephson critical current on temperatures indicates unusual behavior as shown in Appendix B, we confirmed that the CPR is always sinusoidal in the absence of Zeeman fields.

When we apply a Zeeman field in the $y$ direction, the results for $V_{y} / m_{z}=0.004$ and -0.002 in Fig. 2(a) deviate from the sinusoidal relation. A $\varphi$ junction is realized by applying a Zeeman field in the $y$ direction. We also find that the amplitude of the critical current is insensitive to $V_{y}$. The phase shift $\varphi$ in the CPR $J=J_{c} \sin (\theta-\varphi)$ is plotted as a function of $V_{y}$ in Fig. 2(b) with symbols. The results suggest a linear relationship 
(a)

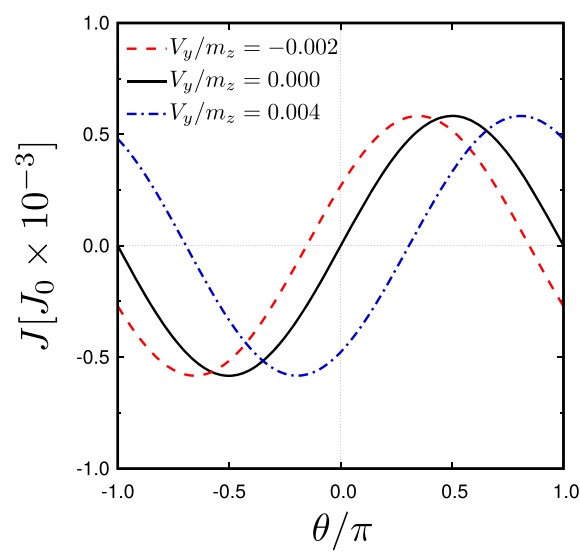

(b)

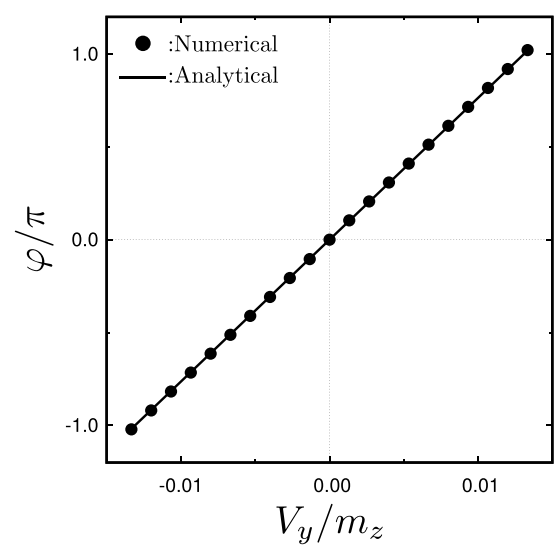

(c)

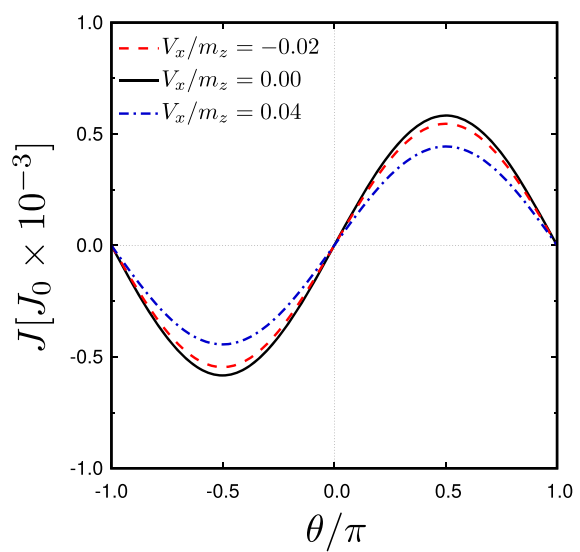

FIG. 2. (a) Josephson current as a function of $\theta$ at $V_{x}=0$. (b) The relation between the phase shift $\varphi$ and $V_{y}$. The results obtained in numerical simulation are shown with symbols. A solid line indicates the analytical results in Eq. (39), where we use $\lambda=t$ and $L=80$ consistently with the numerical simulation. (c) Josephson current as a function of $\theta$ at $V_{y}=0$.

between $\varphi$ and $V_{y}$. Namely, the phase shift $\varphi$ is tunable in a S/QAHI/S junction.

Figure 2(c) shows the numerical results of the Josephson current under Zeeman fields in the $x$ direction. For both $V_{x} / m_{z}=0.04$ and -0.02 , the CPR is sinusoidal. Thus the phase shift depends on the direction of a Zeeman field. These findings are the central results of this paper. In the next section, we will explain the mechanism for the phase shift and its anisotropic response to the Zeeman field by considering the Andreev reflection processes through the chiral edge states in a QAHI.

\section{ORIGIN OF PHASE SHIFT}

\section{A. Symmetry analysis}

To discuss the mechanism for the phase shift $\varphi$ in the CPR, we first analyze the symmetry of Hamiltonian. For this purpose, we describe the Hamiltonian in continuous space as

$$
\begin{gathered}
H_{0}(\theta)=H_{\mathrm{L}}+H_{\mathrm{QAHI}}+H_{\mathrm{R}}, \\
H_{\mathrm{L}(\mathrm{R})}(\boldsymbol{r})=\left[\begin{array}{cc}
\hat{h}(\boldsymbol{r}) & \Delta_{0} i \hat{\sigma}_{2} \mathrm{e}^{i \theta_{\mathrm{L}(\mathrm{R})}} \\
-\Delta_{0} i \hat{\sigma}_{2} \mathrm{e}^{-i \theta_{\mathrm{L}(\mathrm{R})}} & -\hat{h}^{*}(\boldsymbol{r})
\end{array}\right], \\
H_{\mathrm{QAHI}}(\boldsymbol{r})=\left[\begin{array}{cc}
\hat{Q}(\boldsymbol{r}) & 0 \\
0 & -\hat{Q}^{*}(\boldsymbol{r})
\end{array}\right],
\end{gathered}
$$

with

$$
\begin{gathered}
\hat{h}(\boldsymbol{r})=\left(\varepsilon_{\boldsymbol{r}}-\mu_{s}\right) \hat{\sigma}_{0}, \quad \varepsilon_{\boldsymbol{r}}=-\frac{\hbar^{2}}{2 m} \nabla^{2}, \\
\hat{Q}(\boldsymbol{r})=\left(\varepsilon_{\boldsymbol{r}}-m_{z}\right) \hat{\sigma}_{3}+i \lambda \partial_{x} \hat{\sigma}_{2}-i \lambda \partial_{y} \hat{\sigma}_{1} .
\end{gathered}
$$

The Hamiltonian for a Zeeman field is given by

$$
\begin{gathered}
H_{\text {Zeeman }}=H_{V_{x}}+H_{V_{y}}, \\
H_{V_{x}}=\left[\begin{array}{cc}
-V_{x} \hat{\sigma}_{1} & 0 \\
0 & V_{x} \hat{\sigma}_{1}
\end{array}\right], \\
H_{V_{y}}=\left[\begin{array}{cc}
-V_{y} \hat{\sigma}_{2} & 0 \\
0 & -V_{y} \hat{\sigma}_{2}
\end{array}\right] .
\end{gathered}
$$

In the Hamiltonian of a QAHI in Eq. (14), both mirrorreflection symmetry with respect to the $x z$-plane and timereversal symmetry are broken simultaneously. These facts are represented by the relations

$$
\begin{gathered}
\mathcal{M}_{x z} H_{\mathrm{QAHI}}(\boldsymbol{r}) \mathcal{M}_{x z}^{-1} \neq H_{\mathrm{QAHI}}(\boldsymbol{r}), \\
\mathcal{T} H_{\mathrm{QAHI}}(\boldsymbol{r}) \mathcal{T}^{-1} \neq H_{\mathrm{QAHI}}(\boldsymbol{r}), \\
\mathcal{M}_{x z}=\left[\begin{array}{cc}
i \hat{\sigma}_{2} R_{y} & 0 \\
0 & i \hat{\sigma}_{2} R_{y}
\end{array}\right], \quad \mathcal{T}=\left[\begin{array}{cc}
-i \hat{\sigma}_{2} \mathcal{K} & 0 \\
0 & -i \hat{\sigma}_{2} \mathcal{K}
\end{array}\right],
\end{gathered}
$$

where $R_{y}$ is the reflection operator about the $x z$ plane, (i.e., $y \rightarrow-y$ ) and $\mathcal{K}$ donates the complex conjugation. However, the Hamiltonian preserves MMRS with respect to the $x z$ plane, which is defined by combination of $M_{x z}$ and $\mathcal{T}$ as

$$
\begin{gathered}
\mathcal{T}_{x z} H_{\mathrm{QAHI}}(\boldsymbol{r}) \mathcal{T}_{x z}^{-1}=H_{\mathrm{QAHI}}(\boldsymbol{r}), \\
\mathcal{T}_{x z}=\left[\begin{array}{cc}
\hat{\sigma}_{0} R_{y} \mathcal{K} & 0 \\
0 & \hat{\sigma}_{0} R_{y} \mathcal{K}
\end{array}\right],
\end{gathered}
$$

where $\mathcal{T}_{x z}$ is the MMRS operator. By applying $\mathcal{T}_{x z}$ to Eq. (11), we find

$$
\begin{aligned}
& \mathcal{T}_{x z} H_{\mathrm{L}}(\boldsymbol{r}) \mathcal{T}_{x z}^{-1}=\left.H_{\mathrm{L}}(\boldsymbol{r})\right|_{\theta_{L} \rightarrow-\theta_{L}}, \\
& \mathcal{T}_{x z} H_{\mathrm{R}}(\boldsymbol{r}) \mathcal{T}_{x z}^{-1}=\left.H_{\mathrm{R}}(\boldsymbol{r})\right|_{\theta_{R} \rightarrow-\theta_{R}} .
\end{aligned}
$$

As a consequence, we conclude that

$$
\mathcal{T}_{x z} H_{0}(\theta, \boldsymbol{r}) \mathcal{T}_{x z}^{-1}=H_{0}(-\theta, \boldsymbol{r}) .
$$

The Bogoliubov-de Gennes equation can be described as

$$
H_{0}(\theta) \psi_{n}=E_{n}(\theta) \psi_{n},
$$

where $\psi_{n}$ and $E_{n}$ are an eigenstate and an eigenvalue labeled by an index $n$, respectively. By using Eq. (25), the BdG equation can be transformed into

$$
H_{0}(-\theta) \mathcal{T}_{x z} \psi_{n}=E_{n}(\theta) \mathcal{T}_{x z} \psi_{n} .
$$


From Eqs. (26) and (27), we conclude that $H_{0}(\theta)$ and $H_{0}(-\theta)$ have exactly the the same eigenvalues. Namely the relation

$$
E_{n}(\theta)=E_{n}(-\theta) \text {. }
$$

hold true in a S/QAHI/S junction. Generally speaking, the energy of the Josephson junction $F(\theta)$ and the Josephson current are related to each other as

$$
\begin{gathered}
F(\theta)=\sum_{n} E_{n} f_{F}\left(E_{n}\right), \\
J(\theta)=\frac{2 e}{\hbar} \frac{\partial F(\theta)}{\partial \theta},
\end{gathered}
$$

where $f_{F}\left(E_{n}\right)$ is the Fermi distribution function. Due to Eq. (28), the energy of the junction is an even function of the phase difference $\theta$ and the Josephson current is an odd function of $\theta$. Therefore, the CPR satisfies

$$
J(\theta)=-J(-\theta)
$$

which indicates $J(\theta=0)=0$. Thus, MMRS prohibits the appearance of the phase shift $\varphi$ in CPR in the absence of an external Zeeman field.

The effects of the Zeeman field depends on its direction. It is easy to confirm the following relations:

$$
\begin{aligned}
& \mathcal{T}_{x z} H_{V_{x}} \mathcal{T}_{x z}^{-1}=H_{V_{x}}, \\
& \mathcal{T}_{x z} H_{V_{y}} \mathcal{T}_{x z}^{-1} \neq H_{V_{y}} .
\end{aligned}
$$

We find that the Zeeman potential $V_{y}$ breaks MMRS for the $x z$ plane, whereas $V_{x}$ preserves it. As shown in Fig. 2(c), the phase shift is zero even in the presence of $V_{x}$. On the other hand, the phase shift is proportional to $V_{y}$ as shown in Fig. 2(b). The symmetry analysis of the Hamiltonian explains well the anisotropic response of the phase shift to the direction of a Zeeman field. We conclude that breaking MMRS is a necessary condition for realizing a $\varphi$ junction.

The important point of the symmetry analysis can be understood in a more phenomenological way. The relation between the two free energies $F(\theta)$ and $F(-\theta)$ determines the junction property. When $F(\theta)=F(-\theta)$ is satisfied, one can immediately conclude that the junction is either 0 or $\pi$ junction. In the two superconductors, the transformation of $\theta$ to $-\theta$ is realized by applying the complex conjugation to Eq. (11). Therefore, we find $F(\theta)=F(-\theta)$ if $\hat{Q}=\hat{Q}^{*}$ holds in Eq. (14). The Hamiltonian in Eq. (14) satisfies $\hat{Q}^{*}(x, y)=\hat{Q}(x,-y)$ as discussed in Eq. (21). Therefore, the junction may become a $\varphi$ junction in the presence of the potential depending on the $y$ direction. We revisit this issue in Sec. IV.

\section{B. Andreev reflection}

To explain the linear relation between $\varphi$ and $V_{y}$, we analyze the Andreev reflection processes that carry the Josephson current in a S/QAHI/S junction. The chiral edge current flows along two surfaces and two interfaces to the $s$-wave superconductors as illustrated in Fig. 3(a). An electron (a hole) moves in the counterclockwise (clockwise) direction. The direction of spin is locked to the direction of a quasiparticle motion and always points outwardly. The spin-orbit interaction (a)

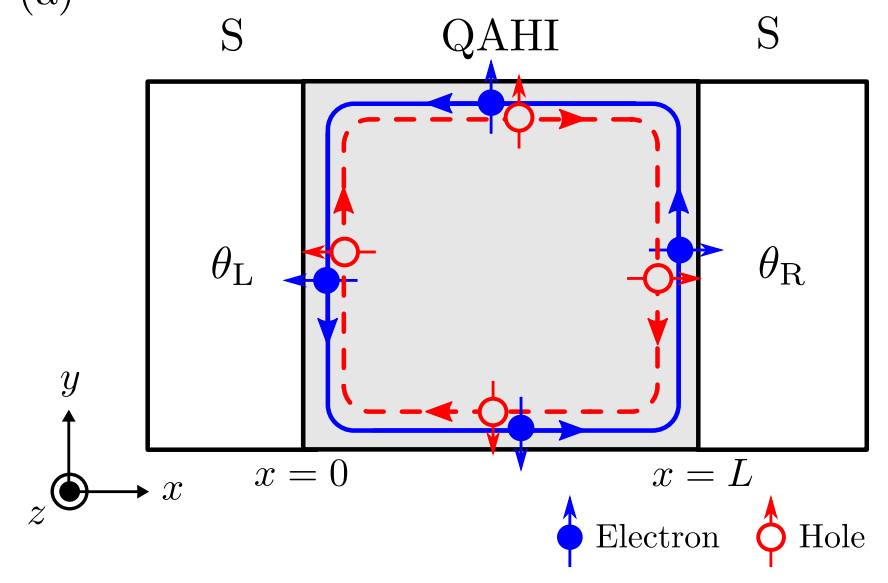

(b)

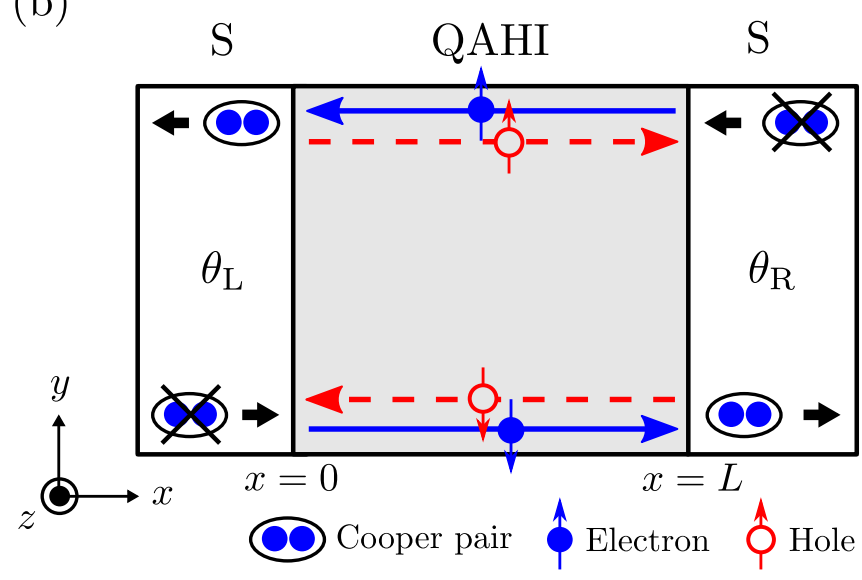

FIG. 3. (a) The chiral edge states in an electron branch and a hole branch. The spin of a quasiparticle always points outside a QAHI. (b) The two Andreev reflection processes contribute to the lowest order Josephson coupling.

changes the spin direction of a quasiparticle at four corners of a QAHI.

When we focus on the edge states at the bottom surface around $y=0$, the pair potential in the superconductors hybridizes an electron and a hole near the junction interfaces at $x=0$ and $L$, which causes the Andreev reflection as shown in Fig. 3(b). The amplitude of the Andreev reflection, however, is expected to be very small due to the spin mismatch in the reflection processes. Usually, a spin-singlet superconductor causes the Andreev reflection, which converts a spin $\uparrow(\downarrow)$ electron into a spin $\downarrow(\uparrow)$ hole. However, at the bottom edge, spin $\uparrow$ channels are absent in both electron and hole branches. In the edge states at the top surface around $y=W$, the spin $\downarrow$ channels are absent. The spin mismatch drastically suppresses the Josephson current. Actually, the amplitude of the Josephson current in Fig. 2 is much smaller than $J_{0}$ even in the absence of potential barrier at the interface. Although we have tried to analyze the spin-flip Andreev reflection process in the presence of spin-orbit coupling, we cannot derive a simple analytic expression of the Andreev reflection coefficients. The numerical results, however, suggest the presence of spin-flip Andreev reflection at the interfaces. Therefore, we assume the 
(a)
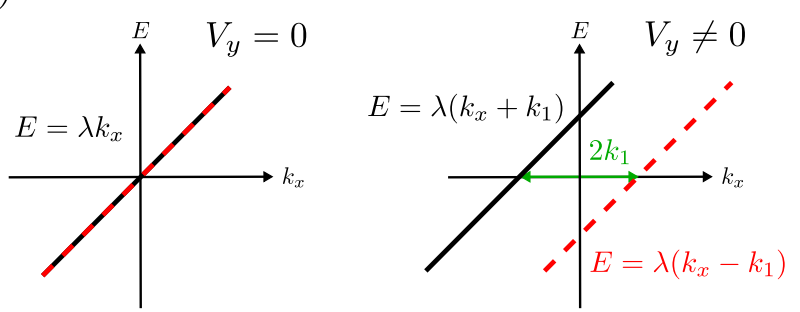

(b)
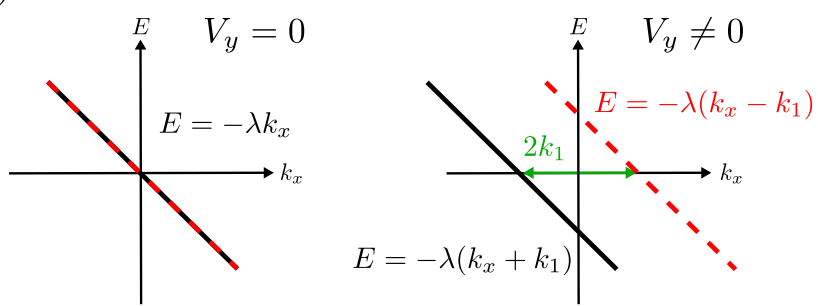

FIG. 4. The dispersion relation of the edge state at the bottom (a) and at the top (b). Solid and broken lines are the dispersion in an electron branch and a hole branch, respectively.

spin-flip Andreev reflection at the two interfaces and describe the phase shift of a quasiparticle in the reflection processes.

Figure 3(b) shows two Andreev reflection processes, which contribute to the lowest order Josephson coupling. In the bottom edge, an electron moves to the right and a hole moves to the left, carrying a Cooper pair from the left superconductor to the right superconductor. At the top edge, a hole moves to the right and an electron moves to the left. Such processes carry a Cooper pair from the right superconductor to the left. The Josephson current is described by the subtraction of the two reflection processes. A current formula [33] may describe the Josephson current in such a situation phenomenologically,

$$
J=\frac{i e}{\hbar} T \sum_{\omega_{n}}\left[r_{L}^{e h} \cdot t_{B}^{h} \cdot r_{R}^{h e} \cdot t_{B}^{e}-\hat{r}_{L}^{h e} \cdot t_{T}^{e} \cdot r_{R}^{e h} \cdot t_{T}^{h}\right]
$$

where $r_{\mathrm{L}(\mathrm{R})}^{h e}\left(r_{\mathrm{L}(\mathrm{R})}^{e h}\right)$ is the spin-flip Andreev reflection coefficient at the left (right) interface from the electron branch to the hole branch (from the hole branch to the electron branch) and $t_{\mathrm{B}(\mathrm{T})}^{e}\left(t_{\mathrm{B}(\mathrm{T})}^{h}\right)$ is the transmission coefficient of an electron (a hole) along the bottom (top) edge. The first (second) term corresponds to the Andreev reflection process at the bottom (top) edge. The Andreev reflection coefficients are phenomenologically described by

$$
\begin{aligned}
& r_{\mathrm{L}(\mathrm{R})}^{h e}=-i \frac{\Delta}{\Omega} \sqrt{t_{I}} e^{-i \theta_{\mathrm{L}(\mathrm{R})}}, \\
& r_{\mathrm{L}(\mathrm{R})}^{e h}=-i \frac{\Delta}{\Omega} \sqrt{t_{I}} e^{i \theta_{\mathrm{L}(\mathrm{R})}},
\end{aligned}
$$

where $t_{I}$ is the effective transmission probability with spin flipping at the interface, and $\Omega=\sqrt{\omega_{n}^{2}+\Delta^{2}}$.

The dispersion relation of the edge state at the bottom and at the top are shown in Figs. 4(a) and 4(b), respectively. The results are analytically obtained by solving the BdG Hamiltonian near the edges. See also Appendix A for details.
In the absence of $V_{y}$, the dispersion in the electron branch and in the hole branch are identical to each other. The wave number in the $x$ direction at the Fermi level is zero. Thus the transmission coefficients would be described by $t_{B}^{e}=$ $t_{B}^{h}=t_{T}^{e}=t_{T}^{h}=t_{0}$ with $t_{0}$ being a real number. The resulting Josephson current in Eq. (34) at $T=0$ becomes

$$
J=\frac{e \Delta}{\hbar} t_{0}^{2} t_{I} \sin \theta .
$$

When we introduce the Zeeman potential in the $y$ direction, the electron dispersion becomes $E=\lambda\left(k_{x}+k_{1}\right)$, whereas the hole dispersion becomes $E=\lambda\left(k_{x}-k_{1}\right)$ at the bottom edge as shown in Fig. 4(a). As shown in Fig. 4(b), the electron and hole dispersions at the top edge are deformed as $E=-\lambda\left(k_{x}+k_{1}\right)$ and $E=-\lambda\left(k_{x}-k_{1}\right)$, respectively. As a consequence, the transmission coefficients through the edge state should be modified as $t_{B}^{e}=t_{0} e^{-i k_{1} L}, t_{B}^{h}=t_{0} e^{-i k_{1} L}, t_{T}^{e}=$ $t_{0} e^{i k_{1} L}, t_{T}^{h}=t_{0} e^{i k_{1} L}$. The Josephson current in Eq. (34) in such a situation becomes

$$
\begin{gathered}
J=\frac{e \Delta}{\hbar} t_{0}^{2} t_{I} \sin (\theta-\varphi), \\
\varphi=2 k_{1} L=\frac{2 V_{y} L}{\lambda} .
\end{gathered}
$$

The phenomenological argument explains the linear relation between the phase shift $\varphi$ on the Zeeman potential $V_{y}$. In Fig. 2(b), we plot $\varphi$ in Eq. (39) with a solid line. The phenomenological results in Eq. (39) explain the numerical results even quantitatively. A Zeeman field in the $y$ direction affects mainly the wave numbers at the edge states. As a result, the amplitude of the Josephson current is independent of $V_{y}$ as shown in Eq. (39) and in numerical simulation in Fig. 2(a). The perfect agreement between Eq. (39) and the numerical results suggests the validity of the phenomenological argument.

\section{ANOTHER $\varphi$ JUNCTION}

The symmetry analysis in Sec. III suggests that the breakdown of MMRS is a trigger of a $\varphi$ junction. To check the validity of the conclusion, we study the effects of breaking MMRS by other physical sources such as (i) random impurity potential in a QAHI and (ii) asymmetric junction geometry with respect to the $x z$ plane. In this section, we set the Zeeman potentials to be zero as $V_{x}=V_{y}=0$.

\section{A. Impurity potential}

The impurity potentials in a QAHI $(1 \leqslant j \leqslant L)$ are considered through the random on-site potential $V_{\text {imp }}(\boldsymbol{r}) \delta_{\boldsymbol{r}, \boldsymbol{r}^{\prime}}$, where $V_{\text {imp }}(\boldsymbol{r})$ is given randomly in the range of $-V_{D} / 2 \leqslant$ $V_{\text {imp }}(\boldsymbol{r}) \leqslant V_{D} / 2$. Equation (7) is transformed as $\hat{Q}(\boldsymbol{r}) \rightarrow$ $\hat{Q}(\boldsymbol{r})+V_{\text {imp }}(\boldsymbol{r}) \hat{\sigma}_{0}$. The impurity potential breaks MMRS because

$$
R_{y} V_{\text {imp }}(x, y) R_{y}^{-1}=V_{\text {imp }}(x,-y) \neq V_{\text {imp }}(x, y),
$$

due to its random character. In Fig. 5, we show the CPR of the Josephson current in the presence of impurity potential with $V_{D}=0.25 t$. The four CPRs with broken lines correspond to the results for four samples with different random impurity 


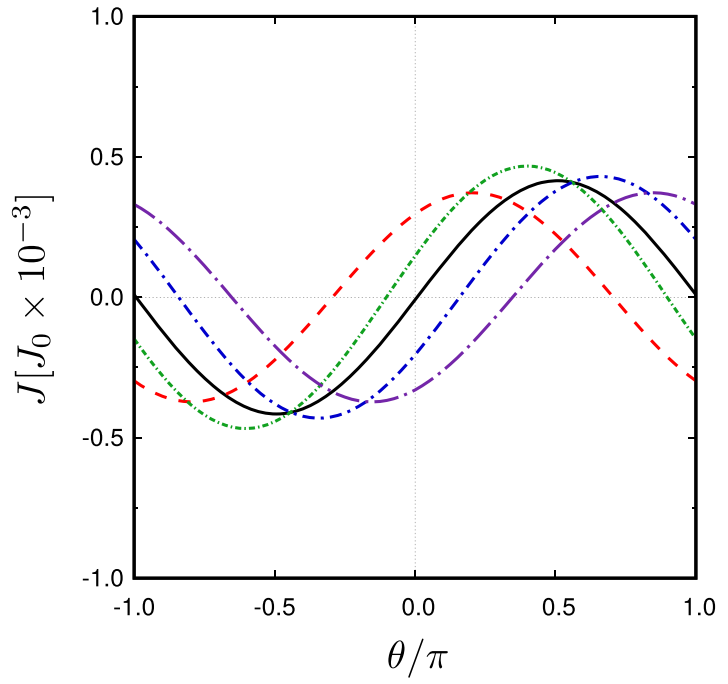

FIG. 5. The Josephson currents for four samples with different impurity configurations are plotted as a function of $\theta$ with broken lines. The ensemble average of the Josephson current over 1500 samples is shown with a solid line.

configurations. The Josephson current flows at $\theta=0$ in all the samples. Although the amplitude of the current is insensitive to the impurity configuration, the phase shift $\varphi_{i}$ for the $i$ th sample depends seriously on the random potential configuration. We also plot the ensemble average of the Josephson current over 1500 samples with a solid line. The results show that the Josephson current, after averaging, recovers the sinusoidal CPR. In experiments, however, the phase shift $\varphi$ is expected in a measurement of the Josephson current in a single sample because the Josephson effect is a phase-coherent phenomenon. Thus the ensemble averaged Josephson current cannot predict a result of one-shot measurement in a single sample [32].

\section{B. Asymmetric junction geometry}

A junction shown in Fig. 6(a) is asymmetric with regard to the $x z$ plane at $y=0$, where we choose the width of two superconductors as $W_{\mathrm{S}}$ and that of a QAHI as $W_{\mathrm{Q}}$. In such a situation, the junction geometry breaks MMRS because the total Hamiltonian is no longer symmetric under $y \leftrightarrow-y$. In Fig. 6(b), the CPR of the Josephson effect for various $W_{\mathrm{S}}$ is represented at $W_{\mathrm{Q}}=20$. The results for $W_{\mathrm{S}}=14$ and $W_{\mathrm{S}}=12$ show the phase shift in the CPR by the breakdown of MMRS due to the two superconducting lead wires. At $W_{\mathrm{S}}=W_{\mathrm{Q}}$, the phase shift becomes zero as shown with a solid line. Within our numerical simulation, however, we cannot find any systematic relation between $W_{\mathrm{S}} / W_{\mathrm{Q}}$ and the phase shift $\varphi$.

In experiments, it is almost impossible to control both the junction geometry and the impurity configuration. Therefore, the phase shift in the CPR always can be expected in every sample. This feature is peculiar to the Josephson junction consisting of a QAHI.

\section{CONCLUSION}

We have studied the direct-current Josephson effect through a quantum anomalous Hall insulator (QAHI) by using the (a)

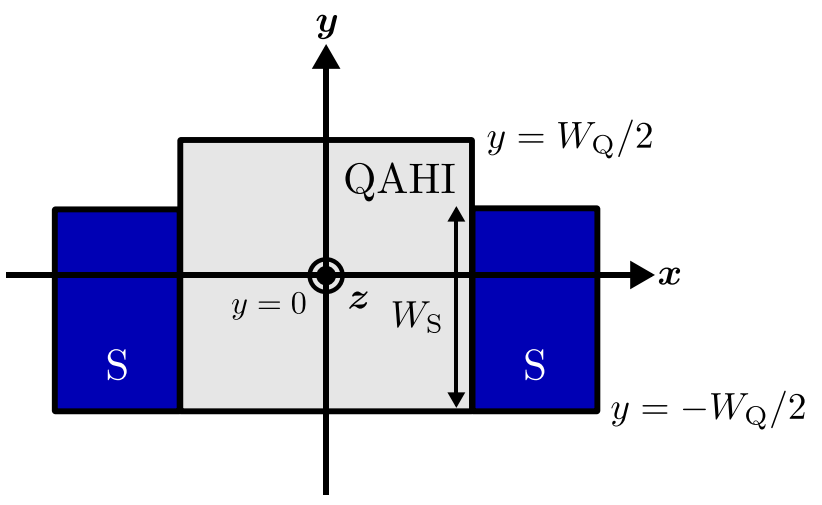

(b)

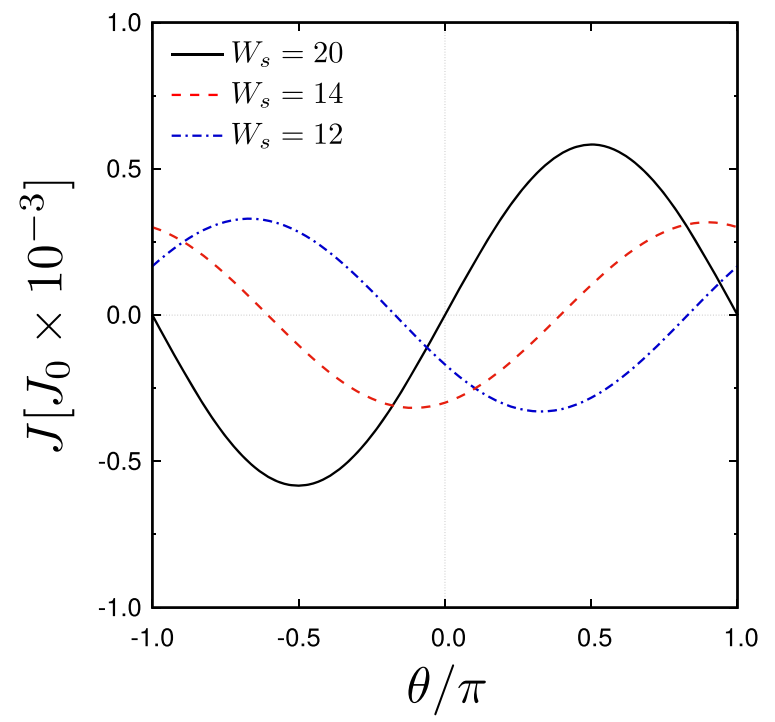

FIG. 6. (a) A Josephson junction asymmetric with respect to the $x z$ plane. The width of the two superconductors $W_{\mathrm{S}}$ is different from that of the QAHI $W_{\mathrm{Q}}$. (b) The Josephson current is plotted as a function of $\theta$ for various $W_{\mathrm{S}}$.

recursive Green function method. A QAHI breaks both time-reversal symmetry and mirror-reflection symmetry simultaneously. However, their combined symmetry, called MMRS, is preserved. The current-phase $(J-\theta)$ relationship (CPR) in the Josephson effect shows very unusual features by reflecting such symmetry property of a QAHI. In the presence of MMRS, the CPR is always sinusoidal as usual, (i.e., $J \propto \sin \theta$ ). In-plane Zeeman fields, random impurities in the QAHI, and asymmetric junction geometries break MMRS. As a consequence, the CPR deviated from the sinusoidal relation to $J \propto(\theta-\varphi)$. The phase shift $\varphi$ by impurities and that by the asymmetric junction geometry would be out of control in experiments. On the other hand, the phase shift is proportional to an in-plane Zeeman field. By considering the Andreev reflection processes phenomenologically, we explain the linear relationship between the phase shift and a Zeeman field. We conclude that $\varphi$ is tunable in a Josephson junction consisting of a QAHI. 


\section{ACKNOWLEDGMENTS}

We are grateful to T. Habe, S.-I. Suzuki, Ya. V. Fominov, and A. A. Golubov for helpful discussion. This paper was supported by Topological Materials Science (No. JP15H05852) and KAKENHI (No. JP15H03525) from the Ministry of Education, Culture, Sports, Science and Technology (MEXT) of Japan, JSPS Core-to-Core Program (A. Advanced Research Networks), Japan-RFBR JSPS Bilateral Joint Research Projects/Seminars (2717G8334b), and by the Ministry of Education and Science of the Russian Federation (Grant No. 14Y.26.31.0007). S.I. is supported in part by a Grant-in-Aid for JSPS Fellows (Grant No. JP16J00956) provided by the Japan Society for the Promotion of Science (JSPS).

\section{APPENDIX A: CHIRAL EDGE STATES}

We represent the analytic expression of wave function and energy dispersion of chiral edge states at a surface of a QAHI in the presence of a Zeeman field. Equation (12) in momentum space is given by

$$
\begin{gathered}
H_{\mathrm{QAHI}}=\left[\begin{array}{cc}
\hat{Q}(\boldsymbol{k}) & 0 \\
0 & -\hat{Q}^{*}(-\boldsymbol{k})
\end{array}\right], \\
\hat{Q}(\boldsymbol{k})=\varepsilon_{\boldsymbol{k}} \hat{\sigma}_{3}-\lambda k_{x} \hat{\sigma}_{2}+\lambda k_{y} \hat{\sigma}_{1}-V_{y} \hat{\sigma}_{2}, \\
\varepsilon_{\boldsymbol{k}}=\frac{\hbar^{2}}{2 m}\left(\boldsymbol{k}^{2}-k_{0}^{2}\right),
\end{gathered}
$$

where $k_{0}=\sqrt{2 m m_{z} / \hbar^{2}}$ is derived from the spontaneous magnetization in the $z$ direction. Since the pair potential is absent, the BdG equation is decoupled into two equations,

$$
\begin{gathered}
\hat{Q}(\boldsymbol{k}) \psi^{e}=E \psi^{e}, \\
-\hat{Q}^{*}(-\boldsymbol{k}) \psi^{h}=E \psi^{h} .
\end{gathered}
$$

In the electron branch, the energy dispersion and the wave functions are obtained as

$$
\left(\begin{array}{c}
E_{\boldsymbol{k}}^{e}+\varepsilon_{\boldsymbol{k}} \\
\lambda\left(k_{y}-i \tilde{k}_{x}\right)
\end{array}\right), \quad\left(\begin{array}{c}
\lambda\left(k_{y}+i \tilde{k}_{x}\right) \\
E_{\boldsymbol{k}}^{e}-\varepsilon_{\boldsymbol{k}}
\end{array}\right),
$$

for $E_{k}^{e}$ and $-E_{k}^{e}$, respectively. Here we define the following quantities:

$$
\begin{gathered}
E_{\boldsymbol{k}}^{e}=\sqrt{\varepsilon_{\boldsymbol{k}}^{2}+\lambda^{2}\left(\tilde{k}_{x}^{2}+k_{y}^{2}\right)}, \\
\tilde{k}_{x}=k_{x}+k_{1}, \quad k_{1}=V_{y} / \lambda .
\end{gathered}
$$

At $V_{y}=0$, the spin-orbit interaction and the magnetic moment induce an energy gap $E_{\mathrm{g}}=2 \lambda k_{0}$. In a weak external Zeeman field $V_{y} \ll m_{z}$, the energy gap remains finite and chiral edge states appear.

To obtain the wave function and the dispersion of the chiral edge state, we consider a semi-infinite system that has a surface perpendicular to the $y$ axis at $y=0$. At an energy $E>0$, the wave function of the edge states $\psi_{\mathrm{Q}}^{e}(y) e^{i k_{x} x}$ is calculated to be

$$
\begin{aligned}
& \psi_{\mathrm{Q}}^{e}(y)=\left(\begin{array}{c}
f_{+} \\
\gamma_{k_{+},-}
\end{array}\right) \mathrm{e}^{i k_{+} y} A_{+}+\left(\begin{array}{c}
\gamma_{-k_{-},+} \\
f_{-}
\end{array}\right) \mathrm{e}^{-i k_{-} y} A_{-}, \\
& f_{ \pm}=E+\sqrt{D^{e}} \mp \lambda k_{0} \tilde{\lambda} / 2, \quad \gamma_{k, \pm}=\lambda\left(k \pm i \tilde{k}_{x}\right),
\end{aligned}
$$

$$
\begin{gathered}
k_{ \pm}=\sqrt{A_{0} \pm\left(2 m / \hbar^{2}\right) \sqrt{D^{e}}}, \\
D^{e}=E^{2}-\lambda^{2}\left\{\left(k_{0}^{2}-k_{x}^{2}+\tilde{k}_{x}^{2}\right\}+\left(\tilde{\lambda} k_{0}\right)^{2} \lambda^{2} / 4,\right. \\
\tilde{\lambda}=\lambda k_{0} / \varepsilon_{0}, \quad \varepsilon_{0}=\hbar^{2} k_{0}^{2} / 2 m, \\
A_{0}=k_{0}^{2}-k_{x}^{2}-\left(\tilde{\lambda} k_{0}\right)^{2} / 2,
\end{gathered}
$$

where $A_{ \pm}$are the amplitudes of the wave function. The condition $D^{e}<0$ results in the complex wave number in the $y$ direction. By imposing the boundary condition $\psi_{Q}^{e}(0)=0$, we obtain the dispersion of chiral edge state as

$$
E_{\mathrm{BS}}^{e}=\lambda\left(k_{x}+k_{1}\right)
$$

for $k_{x}^{2}<k_{0}^{2}\left(1-\tilde{\lambda}^{2} / 4\right)$ and the wave function as

$$
\begin{gathered}
\psi_{\mathrm{ES}}^{e}(y)=C_{0} \mathrm{e}^{-y / \xi} \sin y \sqrt{k_{0}^{2}-k_{x}^{2}}\left(\begin{array}{l}
\delta_{0} \\
\delta_{0}^{*}
\end{array}\right), \\
\xi=\frac{\hbar^{2}}{m \lambda},
\end{gathered}
$$

where $\delta_{0}=\mathrm{e}^{i \pi / 4}$ and $\xi$ is a localization length of the edge states. We have used a relation $\tilde{\lambda} \ll 1$. The dispersion and the wave function in the hole branch can be obtained in a similar way as

$$
\begin{gathered}
E_{\mathrm{BS}}^{h}=\lambda\left(k_{x}-k_{1}\right), \\
\psi_{\mathrm{ES}}^{h}(y)=C_{0} \mathrm{e}^{-y / \xi} \sin y \sqrt{k_{0}^{2}-k_{x}^{2}}\left(\begin{array}{c}
\delta_{0}^{*} \\
\delta_{0}
\end{array}\right),
\end{gathered}
$$

for $k_{x}^{2}<k_{0}\left(1-\tilde{\lambda}^{2} / 4\right)$.

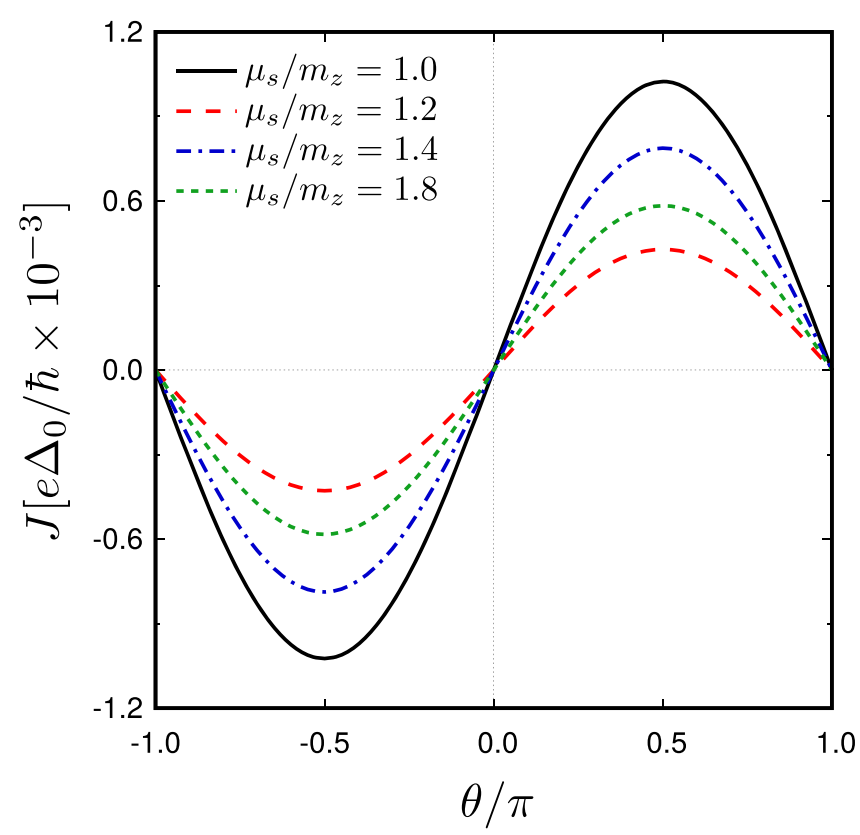

FIG. 7. The Josephson current versus $\theta$ for several choices of $\mu_{s}$ at $T=0.1 T_{c}$. 


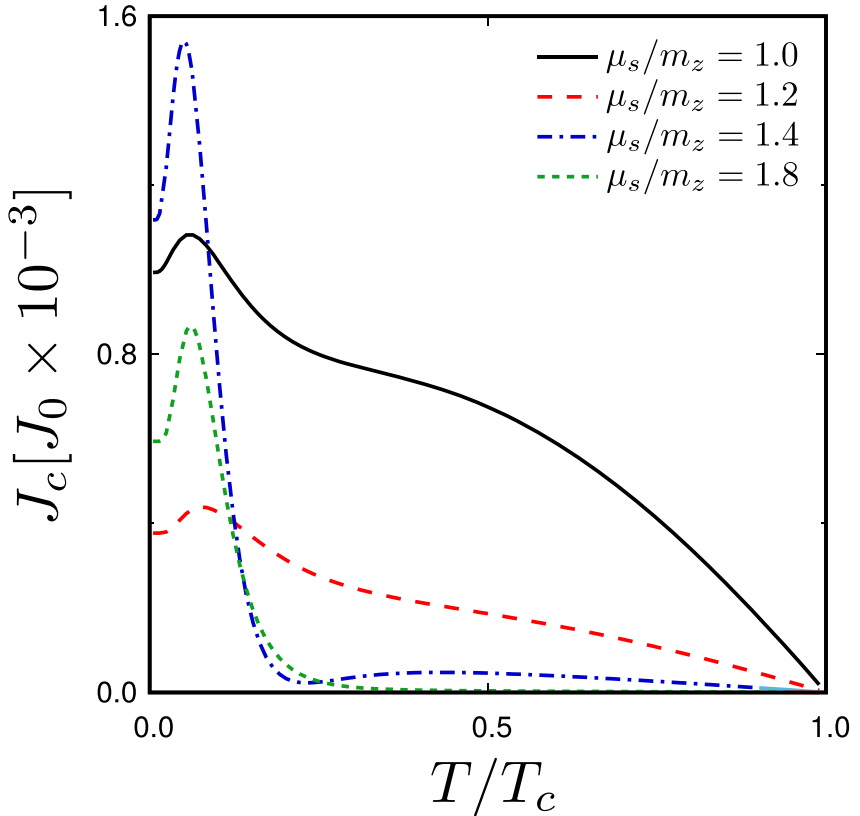

FIG. 8. The critical current $J_{c}$ versus temperature $T$ for various chemical potentials.

The currents of the probability density in the $x$ direction are represented by

$$
\begin{gathered}
J_{x}^{e}=\frac{\lambda}{\hbar} C_{0}^{2} \sin ^{2} y \sqrt{k_{0}^{2}-k_{x}^{2}} \exp ^{-2 y / \xi}, \\
J_{x}^{h}=-\frac{\lambda}{\hbar} C_{0}^{2} \sin ^{2} y \sqrt{k_{0}^{2}-k_{x}^{2}} \exp ^{-2 y / \xi},
\end{gathered}
$$

for an electron and a hole, respectively. Thus, an electron (a hole) moves to the right (left) direction at the bottom edge of a QAHI as shown in Fig. 3.

\section{APPENDIX B: JOSEPHSON CURRENT IN THE ABSENCE OF ZEEMAN FIELD}

We display numerical results of a Josephson current in the absence of Zeeman field $\left(V_{x}=V_{y}=0.0\right)$ to discuss an unusual Josephson effect in a S/QAHI/S junction. As we explained in the text, several parameters are also fixed in

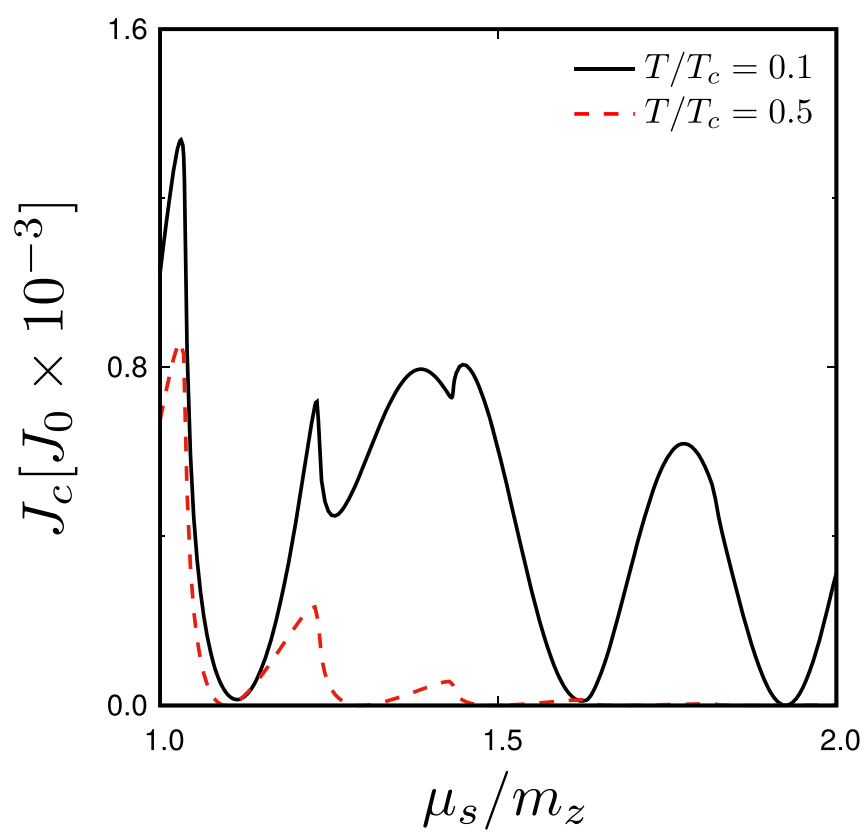

FIG. 9. The critical current versus $\mu_{s}$ for $T=0.1 T_{c}$ and $T=$ $0.5 T_{c}$.

this Appendix as $W=20, L=80, m_{z}=1.5 t, \lambda=1.0 t$, and $\Delta_{0}=0.001 t$.

In Fig. 7, we plot the Josephson current as a function of $\theta$ for several choices of $\mu_{s}$. In all cases, the CPR at $T=0.1 T_{c}$ is sinusoidal in the absence of the Zeeman field.

Unusual features can be seen in the dependence of the Josephson critical current on temperatures as shown in Fig. 8. In all cases, the CPR is sinusoidal as discussed in Fig. 7. The critical current shows the nonmonotonic dependence on temperatures and takes its maximum around $T \approx 0.08 T_{c}=\epsilon_{0}$. The results suggest the existence of a resonantlike state at $\epsilon_{0}$. Unfortunately, however, we cannot figure out physical reasons of such subgap states at the edges of a QAHI. The results for $\mu_{s} / m_{z}=1.4$ and those for 1.8 have a minimum around $T=0.2 T_{c}$. At present, the reasons for such unusual behavior are an open question.

Figure 9 shows the critical current versus $\mu_{s}$. The results for $T=0.1 T_{c}$ correspond to the resonantlike peak in Fig. 8 . At a low temperature $T=0.1 T_{c}$, the results show an aperiodic oscillating behavior as a function of $\mu_{s}$ and become almost zero around $\mu_{s} / m_{z}=1.1,1.6$, and 1.9. The results also suggest existence of a resonantlike subgap states at the edge of a QAHI.
[1] B. D. Josephson, Phys. Lett. 1, 251 (1962).

[2] K. K. Likharev, Rev. Mod. Phys. 51, 101 (1979).

[3] A. A. Golubov, M. Y. Kupriyanov, and E. Ilichev, Rev. Mod. Phys. 76, 411 (2004).

[4] V. Ambegaokar and A. Baratoff, Phys. Rev. Lett. 10, 486 (1963).

[5] A. I. Buzdin, L. N. Bulaevskii, and S. V. Panyukov, JETP Lett. 35, 179 (1982).

[6] A. I. Buzdin, Rev. Mod. Phys. 77, 935 (2005).
[7] V. V. Ryazanov, V. A. Oboznov, A. Yu. Rusanov, A. V. Veretennikov, A. A. Golubov, and J. Aarts, Phys. Rev. Lett. 86, 2427 (2001).

[8] T. Kontos, M. Aprili, J. Lesueur, F. Genet, B. Stephanidis, and R. Boursier, Phys. Rev. Lett. 89, 137007 (2002); A. N. Rossolenko, M. Khabipov, D. Balashov, A. B. Zorin, P. N. Dmitriev, V. P. Koshelets, and A. V. Ustinov, Nat. Phys. 6, 593 (2010). 
[9] S. Kawabata, Y. Asano, Y. Tanaka, A. A. Golubov, and S. Kashiwaya, Phys. Rev. Lett. 104, 117002 (2010).

[10] J.-F. Liu and K. S. Chan, Phys. Rev. B 82, 125305 (2010).

[11] T. Yokoyama, M. Eto, and Y. V. Nazarov, Phys. Rev. B 89, 195407 (2014).

[12] A. Rasmussen, J. Danon, H. Suominen, F. Nichele, M. Kjaergaard, and K. Flensberg, Phys. Rev. B 93, 155406 (2016).

[13] Y. Asano, Y. Sawa, Y. Tanaka, and A. A. Golubov, Phys. Rev. B 76, 224525 (2007).

[14] D. M. Heim, N. G. Pugach, M. Y. Kupriyanov, E. Goldobin, D. Koelle, and R. Kleiner, J. Phys.: Condens. Matter 25, 215701 (2013).

[15] A. A. Reynoso, G. Usaj, C. A. Balseiro, D. Feinberg, and M. Avignon, Phys. Rev. Lett. 101, 107001 (2008).

[16] L. Dell'Anna, A. Zazunov, R. Egger, and T. Martin, Phys. Rev. B 75, 085305 (2007).

[17] A. Zazunov, R. Egger, T. Jonckheere, and T. Martin, Phys. Rev. Lett. 103, 147004 (2009).

[18] A. Brunetti, A. Zazunov, A. Kundu, and R. Egger, Phys. Rev. B 88, 144515 (2013).

[19] G. Campagnano, P. Lucignano, D. Giuliano, and A. Tagliacozzo, J. Phys.: Condens. Matter 27, 205301 (2015).

[20] Y. Tanaka, T. Yokoyama, and N. Nagaosa, Phys. Rev. Lett. 103, 107002 (2009).

[21] F. Dolcini, M. Houzet, and J. S. Meyer, Phys. Rev. B 92, 035428 (2015).
[22] A. Buzdin, Phys. Rev. Lett. 101, 107005 (2008).

[23] D. B. Szombati, S. Nadj-Perge, D. Car, S. R. Plissard, E. P. A. M. Bakkers, and L. P. Kouwenhoven, Nat. Phys. 12, 568 (2016).

[24] C.-Z. Chang, J. Zhang, X. Feng, J. Shen, Z. Zhang, M. Guo, K. Li, Y. Ou, P. Wei, L.-L. Wang, Z.-Q. Ji, Y. Feng, S. Ji, X. Chen, J. Jia, X. Dai, Z. Fang, S.-C. Zhang, K. He, Y. Wang, L. Lu, X.-C. Ma, and Q.-K. Xue, Science 340, 167 (2013).

[25] X. Kou, S.-T. Guo, Y. Fan, L. Pan, M. Lang, Y. Jiang, Q. Shao, T. Nie, K. Murata, J. Tang, Y. Wang, L. He, T.-K. Lee, W.-L. Lee, and K. L. Wang, Phys. Rev. Lett. 113, 137201 (2014).

[26] J. G. Checkelsky, R. Yoshimi, A. Tsukazaki, K. S. Takahashi, Y. Kozuka, J. Falson, M. Kawasaki, and Y. Tokura, Nat. Phys. 10, 731 (2014).

[27] A. J. Bestwick, E. J. Fox, X. Kou, L. Pan, K. L. Wang, and D. Goldhaber-Gordon, Phys. Rev. Lett. 114, 187201 (2015).

[28] C.-Z. Chang, W. Zhao, D. Y. Kim, H. Zhang, B. A. Assaf, D. Heiman, S.-C. Zhang, C. Liu, M. H. W. Chan, and J. S. Moodera, Nat. Mater. 14, 473 (2015).

[29] X.-L. Qi, Y.-S. Wu, and S.-C. Zhang, Phys. Rev. B 74, 085308 (2006).

[30] D. J. Thouless, M. Kohmoto, M. P. Nightingale, and M. den Nijs, Phys. Rev. Lett. 49, 405 (1982).

[31] A. Furusaki, Phys. B (Amsterdam) 203, 214 (1994).

[32] Y. Asano, Phys. Rev. B 63, 052512 (2001).

[33] Y. Asano, Phys. Rev. B 64, 224515 (2001).

[34] P. A. Lee and D. S. Fisher, Phys. Rev. Lett 47, 882 (1981). 